\title{
A Multiperiod Vehicle Lease Planning for Urban Freight Consolidation Network
}

\author{
Woosuk Yang, ${ }^{1}$ Taesu Cheong, ${ }^{2}$ and Sang Hwa Song ${ }^{3}$ \\ ${ }^{1}$ Korea Investment \& Securities, Seoul 1507450, Republic of Korea \\ ${ }^{2}$ School of Industrial Management Engineering, Korea University, Seoul 136713, Republic of Korea \\ ${ }^{3}$ Graduate School of Logistics, Incheon National University, Incheon 406130, Republic of Korea
}

Correspondence should be addressed to Sang Hwa Song; songsh@incheon.ac.kr

Received 26 March 2015; Revised 29 July 2015; Accepted 30 July 2015

Academic Editor: David Bigaud

Copyright (C) 2015 Woosuk Yang et al. This is an open access article distributed under the Creative Commons Attribution License, which permits unrestricted use, distribution, and reproduction in any medium, provided the original work is properly cited.

\begin{abstract}
This paper considers a multiperiod vehicle lease planning problem for urban freight consolidation centers (UFCCs) in the urban freight transport network where short-term-leased and long-term-leased vehicles are hired together. The objective is to allocate the two kinds of leased vehicles optimally for direct transportation services from the associated origin node to the associated UFCC or from the associated UFCC to the associated destinations so as to satisfy a given set of period-to-period freight demands over a given planning horizon at total minimum vehicle allocation cost subject to demand-dependent transportation time restriction. The problem is formulated as an integer programming model and proven to be NP-hard in a strong sense. Thus, a Lagrangian heuristic is proposed to find a good solution efficiently. Numerical experiments show that the proposed algorithm finds good lower and upper bounds within reasonable time.
\end{abstract}

\section{Introduction}

Recent worldwide economic growth has accelerated urbanization, and people have been moving to cities for better jobs and more fulfilling lifestyle. In the late 2000 s, it was reported that more people lived in cities than in rural areas, and it is expected that more people will move to urban areas in the near future $[1,2]$. According to Blanco and Fransoo [3], the number of megacities with at least 10 million people is increasing, and in a decade, their contribution to world economy will steadily grow and constitute more than $20 \%$ of world GDP. As people start to live in concentrated and sometimes congested areas, traffic jams and air pollution continue to increase. As a result, many researchers have started to investigate the impacts of congestion due to urban transportation systems $[2,4]$.

Freight transportation is one of the key contributors to traffic congestion and harmful pollutants in cities. Unlike typical passenger cars, freight vehicles are bigger in size and move more frequently due to the nature of their business.
Furthermore, a recent trend toward just-in-time delivery makes the problem even worse [5]. To survive in competition and to meet increased customer expectation about timely delivery, companies have dispatched small packages more frequently to customers inside metropolitan areas. As average vehicle utilization went down and the number of vehicles dispatched increased, traffic congestion and the subsequent problems have been exacerbated to such an extent that they could cause serious social issues. To deal with the problems caused by urban freight transportation, integrated approaches for city logistics systems have been proposed. Crainic et al. [5] proposed an integrated short-term scheduling of operations management of logistics resources. Ehmke [1] and Ehmke et al. [6] proposed efficient routing systems integrating traffic information and logistics. Yang et al. [7] investigated a problem of designing a city logistics network considering green-house gas emissions. Thus, the research goal of urban freight transportation and city logistics should be aligned with congestion and pollution reduction with less degradation of the city center commercial activities. In this 
regard, multitier urban freight transportation system with consolidation and in-out synchronization, so-called urban freight consolidation, has been proposed as an alternative transportation infrastructure for city logistics.

Urban freight consolidation center (UFCC) is a logistics facility located at the boundary of urban areas to serve freight transportation to city centers including retailers, buildings, and construction sites [8]. Products are transported from their origins such as manufacturers to their destinations in cities through urban consolidation centers. They are first moved to urban consolidation centers, where incoming shipments are unloaded, sorted, and consolidated with other products from different origins. Sorted and consolidated products are then transferred directly to outbound vehicles without storage. In just-in-time delivery environment, it is quite important to send orders to customers when necessary, even though shipment size is not enough to fill a full truck load. By consolidating products in an intermediate point between origins and destinations, urban consolidation centers can contribute to an increase of vehicle utilization, an increase of average size of vehicles involved, and the reduction of delivery frequency. In addition to this, the usage of environmentally friendly vehicles such as electric and clean natural gas power vehicles could often contribute to the decrease of overall harmful emissions from freight transportation vehicles. By improving the overall loading factor of a vehicle destined for congested city centers, urban consolidation centers could effectively reduce the total travel distance and further reduce the impact of freight operations on traffic congestion [9]. In fact, the concept of UFCC has been tested with real business practice in European countries and Toronto, Canada [10-12].

Research on urban freight consolidation centers focuses on the economic analysis of the consolidated freight transportation system. Su and Roorda [12] and Triantafyllou et al. [13] showed that urban consolidation centers can be successfully operated in real urban environments. It was reported that the trial systems were able to reduce harmful emissions and traffic congestion in city centers when properly managed and synchronized. Marcucci and Danielis [8] showed that, in their analysis, urban consolidation centers could attract a considerable amount of freight shipments bound to urban areas. Zhou and Wang [14] studied the issues related to development and construction of consolidation centers and showed that proper strategies based on publicprivate partnership can increase overall economic benefits of the system to participants in city logistics. While the feasibility of urban freight consolidation centers has been studied in depth, operation and network planning issues have been discussed (e.g., [15-17]) but still not been fully investigated yet. The detailed analysis of operation and planning of urban freight consolidation system should be done to be deployed in real business practice. In the supply chain context, an urban consolidation center is similar to the transportation system with cross-docking terminals. In cross-docking network, goods are moved through crossdocking terminals where shipments are sorted, consolidated, and transferred to outbound vehicles in a synchronized manner.
Research on operation and management of the crossdocking based consolidation strategy has progressed in two directions. One is concerned with problems that are related to the internal operations at the consolidation center. Gue [18] analyzed the effects of scheduling trailers into doors on a layout of a freight consolidation center. Bartholdi III and Gue $[19,20]$ designed the layout of a freight consolidation center. Li et al. [21] studied a scheduling problem to minimize storage and order picking in a consolidation center. On the other hand, planning problems on a network level have been considered to locate consolidation centers, to allocate vehicles, and to make vehicle consolidation schedules. Ratliff et al. [22] and Chen et al. [23] considered a problem of making vehicle consolidation schedules for a transportation network. Donaldson et al. [24] considered a problem of allocating vehicles and making vehicle consolidation schedules. Sung and Song [25] and Sung and Yang [26] studied an integrated model of locating consolidation centers and allocating vehicles. All the works on a network level about consolidation based transportation have considered static freight demands, not varying with time. However, freight demands in various industries such as food, apparel, electronic goods, and logistics may be dynamic. Especially in urban freight transportation, demands tend to fluctuate over time. As a cost-efficient way of allocating vehicles to satisfy dynamic freight demands, the issue of vehicle supply on lease has received much research attention, in the situation where the unit-period vehicle lease cost depends on the lease term. The unit-period long-term vehicle lease cost is generally cheaper than the unit-period short-term vehicle lease cost [27-29]. In the case of static freight demands, the long-term vehicle lease is obviously better than the short-term vehicle lease, while in the case of dynamic freight demands, either one does not dominate over the other so that the two lease options need to be considered together. Furthermore, in an urban freight consolidation setting, we need to consider consolidation of shipments which makes the lease planning more difficult.

Therefore, this paper considers a multiperiod vehicle lease planning problem in an urban freight consolidation network (MVLPUC). The problem, denoted by $\mathbb{P}_{\text {MVLPUC }}$, is concerned with optimally allocating the two kinds of leased vehicles for inbound and outbound transportation services so as to satisfy a given set of period-to-period freight demands over a given planning horizon at total minimum vehicle allocation cost subject to demand-dependent transportation time restriction. The planning horizon is divided into discrete time periods such as weeks or months. It is assumed that each freight demand is transported through a single path via one urban consolidation center (where the operations of sorting and consolidating are handled) located between origin and destination nodes, and each outbound vehicle at each urban consolidation center departs as soon as all the associated inbound vehicles arrive and the associated freight demands are sorted appropriately. It is also assumed that an unlimited number of homogeneous capacitated vehicles can be acquired through either long-term lease or short-term lease, with the unit-period short-term vehicle lease cost being greater than or equal to the unit-period long-term vehicle lease cost. 
This paper is organized as follows: Section 2 introduces the problem formulation for $\mathbb{P}_{\text {MVLPUC }}$. In Section 3, we discuss the procedure for solving $\mathbb{P}_{\text {MVLPUC }}$ based on Lagrangian relaxation and problem decompositions. We present the numerical experiments in Section 4 and then conclude our discussion in Section 5.

\section{Model}

In this section, we present the mathematical formulation for Problem $\mathbb{P}_{\text {MVLPUC. }}$. Before introducing the model formulation, all the parameters and decision variables used in this paper are given as follows.

\section{Sets and Parameters}

$\mathscr{J}, \mathscr{J}, \mathscr{K}, \mathscr{V}:$ sets of origin nodes representing manufacturers, destination nodes representing retailers, intermediate nodes representing UFCCs, and all nodes (i.e., $\mathscr{V}=\mathscr{I} \cup \mathscr{K} \cup \mathscr{J}$ ), respectively.

$\mathscr{E}_{\text {IN }}, \mathscr{E}_{\text {OUT }}:$ sets of edges representing potential direct services from origin nodes to intermediate nodes and from intermediate nodes to destination nodes, respectively $\left(\mathscr{E}_{\mathrm{IN}}=\{e=(i, k) \mid i \in \mathscr{I}, k \in \mathscr{K}\}\right.$ and $\left.\mathscr{E}_{\text {OUT }}=\{e=(k, j) \mid k \in \mathscr{K}, j \in \mathscr{J}\}\right)$.

$\mathscr{E}$ : set of all edges (i.e., $\mathscr{E}=\mathscr{E}_{\text {IN }} \cup \mathscr{E}_{\text {OUT }}$ ).

Q: set of freight demands, defined by an ordered pair of two nodes $(i, j)$ for all $i \in \mathscr{I}$ and $j \in \mathscr{J}$.

$Q_{k}$ : subset of $\mathcal{Q}$, each demand of which can be transported through the intermediated node $k \in \mathscr{K}$ within the associated transportation time restriction (to be explained later).

$\mathscr{T}$ : set of time periods.

$d_{i j p}$ : quantity of freight demand $(i, j) \in \mathbb{Q}$ at time period $p \in \mathscr{T}$.

$h_{i k}^{L}, h_{k j}^{L}$ : unit-period long-term vehicle lease cost for $(i, k) \in \mathscr{E}_{\text {IN }}$ and $(k, j) \in \mathscr{E}_{\text {OUT }}$, respectively.

$h_{i k p}^{S}, h_{k j p}^{S}$ : unit-period short-term vehicle lease cost for $(i, k) \in \mathscr{E}_{\text {IN }}$ and $(k, j) \in \mathscr{E}_{\text {OUT }}$, respectively, at time period $p \in \mathscr{T}\left(h_{i k}^{L} \leq h_{i k p}^{S}\right.$ and $\left.h_{k j}^{L} \leq h_{k j p}^{S}\right)$.

$\Gamma$ : vehicle capacity.

$t_{i k}, t_{k j}:$ transportation time elapsed for each $(i, k) \in$ $\mathscr{E}_{\text {IN }}$ and $(k, j) \in \mathscr{E}_{\text {OUT }}$, respectively.

$o_{k}$ : handling (sorting and consolidating) time at the intermediate node $k \in \mathscr{K}$.

$\mathrm{TL}_{i j}$ : transportation time restriction required for freight demand $(i, j) \in \mathbb{Q}$.

\section{Decision Variables}

$X_{i j k p}: 1$ if freight demand $(i, j) \in \mathbb{Q}$ is transported in period $p \in \mathscr{T}$ through the intermediate node $k \in \mathscr{K}$ and 0 otherwise.

$Y_{i k}, Y_{k j}:$ numbers of long-term-leased vehicles allocated for $(i, k) \in \mathscr{E}_{\text {IN }}$ and $(k, j) \in \mathscr{E}_{\text {OUT }}$, respectively.
$Z_{i k p}, Z_{k j p}$ : numbers of short-term-leased vehicles allocated for $(i, k) \in \mathscr{E}_{\text {IN }}$ and $(k, j) \in \mathscr{E}_{\text {OUT }}$, respectively, in period $p \in \mathscr{T}$.

We note that, for notational simplicity, notations (or subscripts) $e$ and $(i, k)$ (or $(k, j))$ are used alternately to refer to freight demand $(i, k)$ (or $(k, j))$ on the corresponding edge $e$. Furthermore, the two terms "edge" and "direct service" are used interchangeably in this paper. Then, we now present the problem formulation for Problem $\mathbb{P}_{\text {MVLPUC }}$ as follows.

\section{Problem $\mathbb{P}_{\text {MVLPUC }}$}

$$
\begin{aligned}
\min & \sum_{(i, k) \in \mathscr{E}_{\text {IN }}}\left(|\mathscr{T}| h_{i k}^{L} Y_{i k}+\sum_{p \in \mathscr{T}} h_{i k p}^{S} Z_{i k p}\right) \\
& +\sum_{(k, j) \in \mathscr{E}_{\text {OUT }}}\left(|\mathscr{T}| h_{k j}^{L} Y_{k j}+\sum_{p \in \mathscr{T}} h_{k j p}^{S} Z_{k j p}\right)
\end{aligned}
$$

subject to $\sum_{\left\{k \in \mathscr{K} \mid(i, j) \in \mathbb{Q}_{k}\right\}} X_{i j k p}=1$,

$$
\forall(i, j) \in \mathbb{Q}, \forall p \in \mathscr{T}
$$

$$
\sum_{\left\{j \in \mathcal{F} \mid(i, j) \in \mathbb{Q}_{k}\right\}} d_{i j p} X_{i j k p} \leq \Gamma\left(Y_{i k}+Z_{i k p}\right),
$$

$$
\sum_{\left\{i \in \mathcal{F} \mid(i, j) \in \mathbb{Q}_{k}\right\}} d_{i j p} X_{i j k p} \leq \Gamma\left(Y_{k j}+Z_{k j p}\right)
$$

$$
\forall(k, j) \in \mathscr{E}_{\text {OUT }}, \forall p \in \mathscr{T}
$$

$$
\begin{aligned}
& Y_{i k}, Z_{i k p}, Y_{k j}, Z_{k j p} \in \mathbb{Z}^{+}, \\
& X_{i j k p} \in\{0,1\} .
\end{aligned}
$$

The objective function (1a) represents the cost of allocating any long-term-leased and short-term-leased vehicles for edges. Constraints (1b) imply that all the freight demands have to be serviced for each period. Constraints (1c) and (1d) require that the total amount of demands transported through any edge should not exceed the total capacity of any allocated vehicles for each period. In regard to the set $Q_{k}$ in constraints (1b), (1c), and (1d), we assume that there exists the transportation time limit $\mathrm{TL}_{i j}$ for each freight demand $(i, j)$ such that the sum of the transportation times between nodes and the handling time at an intermediate node $k$ does not exceed $\mathrm{TL}_{i j}$ (i.e., $t_{i k}+o_{k}+t_{k j} \leq \mathrm{TL}_{i j}$ ). Thus, for each $k \in \mathscr{K}$, the elements of set $Q_{k}$ can be identified as freight demands $(i, j)$ in $Q$ that satisfy the aforementioned condition with $\mathrm{TL}_{i j}$ at a preprocessing stage.

\section{Solution Approach}

In this section, we propose a heuristic approach based on Lagrangian relaxation. Problem $\mathbb{P}_{\text {MVLPUC }}$ can be proven as NP-hard in a strong sense in the same manner as 
shown in Sung and Song [25]. Moreover, $\mathbb{P}_{\text {MVLPUC }}$ considers time-varying demands, so it may be too complex to derive an exact algorithm to find optimal solutions even for small-sized problem instances. Therefore, we here propose an efficient heuristic method based on the Lagrangian relaxation.

3.1. Lagrangian Relaxation. Before relaxing constraints in $\mathbb{P}_{\text {MVLPUC }}$ for Lagrangian relaxation heuristic, the following problem $\mathbb{P}_{\text {MVLPUC }_{+}}$is derived by introducing another decision variable $\bar{X}_{i j k p}$ (equivalent to variables $X_{i j k p}$ ) and adding a set of constraints $X_{i j k p} \leq \bar{X}_{i j k p}$ to $\mathbb{P}_{\text {MVLPUC }}$.

Problem $\mathbb{P}_{\mathrm{MVLPUC}_{+}}$

$$
\begin{aligned}
v_{\mathrm{OPT}}=\min & \sum_{(i, k) \in \mathscr{E}_{\mathrm{IN}}}\left(|\mathscr{T}| h_{i k}^{L} Y_{i k}+\sum_{p \in \mathscr{T}} h_{i k p}^{S} Z_{i k p}\right) \\
& +\sum_{(k, j) \in \mathscr{E}_{\text {OUT }}}\left(|\mathscr{T}| h_{k j}^{L} Y_{k j}+\sum_{p \in \mathscr{T}} h_{k j p}^{S} Z_{k j p}\right)
\end{aligned}
$$

subject to $\sum_{\left\{k \in \mathscr{K} \mid(i, j) \in \mathbb{Q}_{k}\right\}} X_{i j k p}=1$,

$$
\begin{gathered}
\sum_{\left\{j \in \mathcal{F} \mid(i, j) \in Q_{k}\right\}} d_{i j p} X_{i j k p} \leq \Gamma\left(Y_{i k}+Z_{i k p}\right), \\
\forall(i, k) \in \mathscr{E}_{\mathrm{IN}}, \forall p \in \mathscr{T} \\
X_{i j k p} \leq \bar{X}_{i j k p}, \quad \\
\forall k \in \mathscr{K}, \forall(i, j) \in Q_{k}, \forall p \in \mathscr{T} \\
\sum_{\left\{i \in \mathcal{F} \mid(i, j) \in Q_{k}\right\}} d_{i j p} \bar{X}_{i j k p} \leq \Gamma\left(Y_{k j}+Z_{k j p}\right), \\
\forall(k, j) \in \mathscr{E}_{\text {OUT }}, \forall p \in \mathscr{T} \\
Y_{i k}, Z_{i k p}, Y_{k j}, Z_{k j p} \in \mathbb{Z}^{+}, \\
X_{i j k p}, \bar{X}_{i j k p} \in\{0,1\} .
\end{gathered}
$$

Problem $\mathbb{P}_{\text {MVLPUC }_{+}}$has some obviously redundant variables $\bar{X}_{i j k p}$ and the associated constraints (2d) while it yields the interesting problem structure which is good to apply the Lagrangian relaxation method. That is, if constraints (2b) and (2d) in $\mathbb{P}_{\text {MVLPUC }_{+}}$are Lagrangian relaxed, then the resulting problem can be decomposed into single-edge problems. Thus, constraints $(2 \mathrm{~b})$ and $(2 \mathrm{~d})$ are Lagrangian relaxed with Lagrange multipliers $\mu_{i j p}^{\prime}$ (unrestricted) and $\mu_{i j k p}^{\prime \prime}(\geq 0)$, respectively. For given $\mu \equiv\left(\mu_{i j p}^{\prime}, \mu_{i j k p}^{\prime \prime}\right)_{\forall i, j, k, p}$, the resulting problem $\mathbb{P}_{\mathrm{LR}_{\mu}}$ can be derived as follows.

Problem $\mathbb{P}_{L R_{\mu}}$

$$
\begin{aligned}
v(\mu)=\min \sum_{(i, j) \in \mathscr{Q}} \sum_{p \in \mathscr{T}} \mu_{i j p}^{\prime}+\sum_{(i, k) \in \mathscr{E}_{\mathbb{N}}}\left[|\mathscr{T}| h_{i k}^{L} Y_{i k}+\sum_{p \in \mathscr{T}}\left(h_{i k p}^{S} Z_{i k p}-\sum_{\left\{j \in \mathcal{F} \mid(i, j) \in \mathbb{Q}_{k}\right\}} \theta_{i j k p} X_{i j k p}\right)\right] \\
+\sum_{(k, j) \in \mathscr{E}_{\text {OUT }}}\left[|\mathscr{T}| h_{k j}^{L} Y_{k j}+\sum_{p \in \mathscr{T}}\left(h_{k j p}^{S} Z_{k j p}-\sum_{\left\{i \in \mathscr{F} \mid(i, j) \in \mathbb{Q}_{k}\right\}} \delta_{i j k p} \bar{X}_{i j k p}\right)\right]
\end{aligned}
$$

subject to $(2 \mathrm{c}),(2 \mathrm{e}),(2 \mathrm{f})$,

$$
\text { where } \theta_{i j k p}=\mu_{i j p}^{\prime}-\mu_{i j k p}^{\prime \prime}, \delta_{i j k p}=\mu_{i j k p}^{\prime \prime} \text {. }
$$

It is evident that $v(\mu)$ is a lower bound on $v_{\mathrm{OPT}}$ for any given $\mu$. Let $v^{*}(\mu)=\max _{\mu} v(\mu)$, and let $v_{\mathrm{LP}}$ be a lower bound (on $v_{\mathrm{OPT}}$ ) obtained by solving the linear programming (LP) relaxation problem of $\mathbb{P}_{\text {MVLPUC. }}$. Then, the proposed Lagrangian relaxation $\mathbb{P}_{\mathrm{LR}_{\mu}}$ provides a good lower bound as indicated in Proposition 1. Since the proposition below is obvious to show, we omit the proof.
Furthermore, as mentioned earlier, Problem $\mathbb{P}_{\mathrm{LR}_{\mu}}$ can be decomposed into single-edge problems of selecting demand and allocating vehicles (SEPDV), resulting in $\mathbb{P}_{\text {SEPDV }_{i k, \mu}}$ and $\mathbb{P}_{\mathrm{SEPDV}_{k, \mu}}$ for each $(i, k) \in \mathscr{E}_{\mathrm{IN}}$ and $(k, j) \in \mathscr{E}_{\text {OUT }}$, respectively, as follows.

Problem $\mathbb{P}_{S E P D V_{i k, \mu}}$

Proposition 1. Consider $v_{\mathrm{OPT}} \geq v^{*}(\mu) \geq v_{L P}$.

$$
v_{\mathrm{SEPDV}}^{i k}(\mu)=\min \quad|\mathscr{T}| h_{i k}^{L} Y_{i k}+\sum_{p \in \mathscr{T}}\left(h_{i k p}^{S} Z_{i k p}-\sum_{\left\{j \in \mathcal{F} \mid(i, j) \in \mathbb{Q}_{k}\right\}} \theta_{i j k p} X_{i j k p}\right)
$$




$$
\begin{array}{ll}
\text { subject to } & \sum_{\left\{j \in \mathcal{F} \mid(i, j) \in \mathbb{Q}_{k}\right\}} d_{i j p} X_{i j k p} \leq \Gamma\left(Y_{i k}+Z_{i k p}\right), \quad \forall p \in \mathscr{T} \\
& Y_{i k}, Z_{i k p} \in \mathbb{Z}^{+}, X_{i j k p} \in\{0,1\}, \quad \forall p \in \mathscr{T} .
\end{array}
$$

\section{Problem $\mathbb{P}_{S E P D V_{k j, \mu}}$}

$$
\begin{array}{ll}
v_{\mathrm{SEPDV}}^{k j}(\mu)=\min \quad|\mathscr{T}| h_{k j}^{L} Y_{k j} & \\
+\sum_{p \in \mathscr{T}}\left(h_{k j p}^{S} Z_{k j p}-\sum_{\left\{i \in \mathscr{F} \mid(i, j) \in \mathbb{Q}_{k}\right\}} \delta_{i j k p} \bar{X}_{i j k p}\right) \\
\text { subject to } \sum_{\left\{i \in \mathcal{F} \mid(i, j) \in \mathbb{Q}_{k}\right\}} d_{i j p} \bar{X}_{i j k p} \leq \Gamma\left(Y_{k j}+Z_{k j p}\right), & \quad \forall p \in \mathscr{T} \\
& Y_{k j}, Z_{k j p} \in \mathbb{Z}^{+}, \bar{X}_{i j k p} \in\{0,1\}, \quad \forall p \in \mathscr{T} .
\end{array}
$$

It is straightforward to show that, for given $\mu$,

$$
\begin{aligned}
v(\mu)= & \sum_{(i, j) \in \mathscr{Q}} \sum_{p \in \mathscr{T}} \mu_{i j p}^{\prime}+\sum_{(i, k) \in \mathscr{E}_{\mathrm{IN}}} v_{\mathrm{SEPDV}}^{i k}(\mu) \\
& +\sum_{(k, j) \in \mathscr{E}_{\mathrm{OUT}}} v_{\mathrm{SEPDV}}^{k j}(\mu)
\end{aligned}
$$

and hence, $v(\mu)$ can be evaluated through $v_{\text {SEPDV }}^{i k}(\mu)$ and $v_{\mathrm{SEPDV}}^{k j}(\mu)$. Since $\mathbb{P}_{\mathrm{SEPDV}_{i k, \mu}}$ and $\mathbb{P}_{\mathrm{SEPDV}_{k j, \mu}}$ have the same problem structure, we only discuss the procedure for solving $\mathbb{P}_{\text {SEPDV }_{i k, \mu}}$ in the next section.

3.2. Solving $\mathbb{P}_{S E P D V_{i k, \mu}}$. In this section, we present the solution procedure for $\mathbb{P}_{\text {SEPDV }_{i k, \mu}}$ so that we can eventually evaluate the value $v(\mu)$ of $\mathbb{P}_{L^{2}}$ for each $\mu$. First, for given $\mu$, Proposition 2 and Corollary 3 are characterized so as to reduce the solution space of $X$ and $(Y, Z)$ variables, respectively, in $\mathbb{P}_{S E P D V}$ ik, .

Proposition 2. For given $\mu$, the following statements hold in $\mathbb{P}_{S E P D V_{i k, \mu}}$ :

(a) If $\theta_{i j k p} \leq 0$ for some $j \in \mathscr{J}$ and $p \in \mathscr{T}$, then there is an optimal solution with $X_{i j k p}=0$.

(b) If $\theta_{i j k p} \geq h_{i k p}^{S}\left\lceil d_{i j p} / \Gamma\right\rceil$ for some $j \in \mathscr{J}$ and $p \in \mathscr{T}$, then there is an optimal solution with $X_{i j k p}=1$.

Proof. (a) Let us compare the situations of $X_{i j k p}=1$ and $X_{i j k p}=0$ for some $j \in \mathscr{J}$ and $p \in \mathscr{T}$ such that $\theta_{i j k p} \leq$ 0 . The value of the objective function (4a) for the former case cannot be smaller than that for the latter case because the coefficient of the $X$ variable in (4a) is nonnegative. Also, the former case makes the constraint (4b) tighter than the latter case. Therefore, the former case (i.e., $X_{i j k p}=1$ ) cannot give a better solution than the latter case (i.e., $X_{i j k p}=0$ ).

(b) This can be shown in the same manner as in the proof of (a), and hence we omit its proof.
Let $Q_{i k}$ be a set of freight demands that can be transported through $(i, k)$ where $i \in \mathscr{I}$ and $k \in \mathscr{K}$. Note that $Q_{k}=\bigcup_{i \in \mathscr{I}} \widetilde{Q}_{i k}$. For $p \in \mathscr{T}$, let $\widetilde{Q}_{i k p}^{0}$ and $\widetilde{Q}_{i k p}^{1}$ be subsets of $Q_{i k}$ satisfying Propositions 2(a) and 2(b) in $\mathbb{P}_{\mathrm{SEPDV}_{i k, \mu}}$, respectively, and let $\widetilde{Q}_{i k p}=\widetilde{Q}_{i k p}^{0} \cup \widetilde{Q}_{i k p}^{1}$.

Corollary 3. For given $\mu$ and $\left\{X_{i j k p}\right\}$ fixed at the associated values according to Proposition 2, there is an optimal solution in $\mathbb{P}_{S E P D V_{i k, \mu}}$ with $\left(Y_{i k}, Z_{i k p}\right) \in\left\{\left(y, z_{p}\right) \mid \tilde{Y}_{i k}^{\text {min }} \leq y \leq\right.$ $\left.\widetilde{Y}_{i k}^{\max }, 0 \leq z_{p} \leq \widetilde{Z}_{i k p, y}^{\max }, \forall p \in \mathscr{T}\right\}$, where

$$
\begin{aligned}
\widetilde{Y}_{i k}^{\min } & =\left[\frac{\min _{p \in \mathscr{T}} \sum_{\left\{j \in \mathscr{F} \mid(i, j) \in \widetilde{Q}_{i k p}^{1}\right\}} d_{i j p}}{\Gamma}\right], \\
\widetilde{Y}_{i k}^{\max } & =\left[\frac{\max _{p \in \mathscr{T}} \sum_{\left\{j \in \mathscr{F} \mid(i, j) \in \mathbb{Q}_{i k} \backslash \widetilde{Q}_{i k p}^{0}\right\}} d_{i j p}}{\Gamma}\right], \\
\widetilde{Z}_{i k p, y}^{\max } & =\left[\frac{\sum_{\left\{j \in \mathscr{F} \mid(i, j) \in \mathbb{Q}_{i k} \backslash \widetilde{Q}_{i k p}^{0}\right\}} d_{i j p}}{\Gamma}\right]-y .
\end{aligned}
$$

Proof. The constraint (4b) can be rewritten as

$$
\begin{aligned}
& \sum_{\left\{j \in \mathcal{F} \mid(i, j) \in \widetilde{Q}_{i k p}^{1}\right\}} d_{i j p}+\sum_{\left\{j \in \mathcal{F} \mid(i, j) \in \mathbb{Q}_{i k} \backslash \widetilde{Q}_{i k p}\right\}} d_{i j p} X_{i j k p} \\
& \leq \Gamma\left(Y_{i k}+Z_{i k p}\right)
\end{aligned}
$$

for all $p \in \mathscr{T}$. Since all the variables are binary variables, $\tilde{Y}_{i k}^{\text {min }} \leq\left\lceil\sum_{\left\{j \in \mathcal{F} \mid(i, j) \in \widetilde{Q}_{i k p}^{1}\right\}} d_{i j p} / \Gamma\right\rceil \leq\left(Y_{i k}+Z_{i k p}\right)$ holds for all $p \in \mathscr{T}$. Since it is assumed that $0<h_{i k}^{L} \leq h_{i k p}^{S}$ for all $p \in \mathscr{T}$, any feasible solution with $\left(X_{i j k p}, Y_{i k}, Z_{i k p}\right)=\left(x_{j p}\right.$, $\left.y, z_{p}\right)$, where $y<\widetilde{Y}_{i k}^{\min }$ cannot yield a better solution value than the feasible solution with $\left(X_{i j k p}, Y_{i k}, Z_{i k p}\right)=\left(x_{j p}, \widetilde{Y}_{i k}^{\mathrm{min}}\right.$, $\left.\max \left\{0, z_{p}-\widetilde{Y}_{i k}^{\min }+y\right\}\right)$. Moveover, any feasible solution with $\left(X_{i j k p}, Y_{i k}, Z_{i k p}\right)=\left(x_{j p}, y, z_{p}\right)$, where $y>\tilde{Y}_{i k}^{\max }$ cannot yield a better solution value than the feasible solution with $\left(X_{i j k p}, Y_{i k}, Z_{i k p}\right)=\left(x_{j p}, \tilde{Y}_{i k}^{\max }, 0\right)$. Therefore, there is an optimal solution with $\tilde{Y}_{i k}^{\min } \leq Y_{i k} \leq \tilde{Y}_{i k}^{\max }$. Also, with $Y_{i k}$ fixed at $y \in\left\{y^{\prime} \in \mathbb{Z}^{+} \mid \widetilde{Y}_{i k}^{\min } \leq y^{\prime} \leq \widetilde{Y}_{i k}^{\max }\right\}$, it is obvious that there is an optimal solution with $0 \leq Z_{i k p} \leq \widetilde{Z}_{i k p, y}^{\max }$.

Then, the restricted problem with $Y_{i k}$ fixed at $y \in\left\{y^{\prime} \in\right.$ $\left.\mathbb{Z}^{+} \mid \tilde{Y}_{i k}^{\min } \leq y^{\prime} \leq \widetilde{Y}_{i k}^{\max }\right\}$ in $\mathbb{P}_{\mathrm{SEPDV}_{i k, \mu}}$, denoted by $\mathbb{P}_{\mathrm{SEPDV}_{i k, \mu, y}}$, can be decomposed into single-period bounded knapsack problems (BKP), $\mathbb{P}_{\mathrm{BKP}_{i k p, \mu, y}}$, as follows. 
Problem $\mathbb{P}_{B K P_{i k p, \mu, y}}$

$$
\begin{aligned}
& v_{\mathrm{BKP}}^{i k p}(\mu, y)=\max \quad h_{i k p}^{S} \bar{Z}_{i k p}+\sum_{\left\{j \in \mathcal{F}\left|(i, j) \in G_{i k}\right| \widetilde{Q}_{i k p}^{0}\right\}} \theta_{i j k p} X_{i j k p} \\
& \text { subject to } \sum_{\left\{j \in \mathscr{F}\left|(i, j) \in \widehat{Q}_{i k}\right| \widetilde{Q}_{i k p}\right\}} d_{i j p} X_{i j k p}+\Gamma \bar{Z}_{i k p} \leq \Gamma\left\lceil\frac{\sum_{\left\{j \in \mathcal{F}\left|(i, j) \in Q_{i k}\right| \widetilde{Q}_{i k p}^{0}\right\}} d_{i j p}}{\Gamma}\right\rceil-\sum_{\left\{j \in \mathcal{F} \mid(i, j) \in \widetilde{Q}_{i k p}^{1}\right\}} d_{i j p} \\
& \bar{Z}_{i k p} \in\left\{0,1, \ldots,\left(\left\lceil\frac{\sum_{\left\{j \in \mathcal{F} \mid(i, j) \in \widehat{Q}_{i k} \widetilde{a}_{i k p}^{0}\right.} d_{i j p}}{\Gamma}\right\rceil-y\right)\right\}, X_{i j k p} \in\{0,1\} .
\end{aligned}
$$

Note that $\bar{Z}_{i k p}=\widetilde{Z}_{i k p, y}^{\max }-Z_{i k p}$. We next discuss how to solve $\mathbb{P}_{\mathrm{BKP}_{i k p, \mu, y}}$. Let $v_{\mathrm{SEPDV}}^{i k}(\mu, y)$ denote the optimal value of

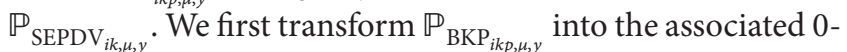
1 knapsack problem, and the knapsack problem can be solved by the dynamic programming algorithm proposed by Toth [30]. We remark that $\bar{Z}_{i k p}$ takes the finite number of integer values in the set $\Psi \equiv\left\{0,1, \ldots,\left(\left\lceil\sum_{\left\{j \in \mathcal{F}\left|(i, j) \in \widehat{Q}_{i k}\right| \widetilde{Q}_{i k p}^{0}\right\}} d_{i j p} / \Gamma\right\rceil-\right.\right.$ $y)\}$. Furthermore, for each value of $\bar{Z}_{i k p}$ in $\Psi$, it is straightforward to show that the problem $\mathbb{P}_{\mathrm{BKP}_{i k p, \mu, y}}$ reduces to a 0 1 knapsack problem with decision variables $X_{i j k p}$ for all $j$. Therefore, we iteratively solve a $0-1$ knapsack problem for each value of $\bar{Z}_{i k p}$ in $\Psi$, compare the objective function values for each case, and then identify the optimal solution for $\mathbb{P}_{\mathrm{BKP}_{i k p, \mu, y}}$. Once all the problems of $\mathbb{P}_{\mathrm{BKP}_{i k p, \mu, y}}$ are solved for each value of $Y_{i k}$ as mentioned above, the optimal value of the problem $\mathbb{P}_{\mathrm{SEPDV}_{i k, \mu}} v_{\mathrm{SEPDV}}^{i k}(\mu)$, is computed as

$$
\begin{aligned}
& v_{\text {SEPDV }}^{i k}(\mu)=\min _{\tilde{Y}_{i k}^{\min } \leq y \leq \widetilde{Y}_{i k}^{\max }} v_{S \operatorname{SEPDV}}^{i k}(\mu, y)=\min _{\tilde{Y}_{i k}^{\min } \leq y \leq \widetilde{Y}_{i k}^{\max }}[|\mathscr{T}| \\
& \cdot h_{i k}^{L} y \\
& \left.+\sum_{p \in \mathscr{T}}\left(h_{i k p}^{S} \widetilde{Z}_{i k p, y}^{\max }-v_{\mathrm{BKP}}^{i k p}(\mu, y)-\sum_{\left\{j \in \mathcal{F} \mid(i, j) \in \widetilde{G}_{i k p}^{1}\right\}} \theta_{i j k p}\right)\right] .
\end{aligned}
$$

The number of problems $\mathbb{P}_{\mathrm{BKP}_{i k p, \mu, y}}$ and $\mathbb{P}_{\mathrm{SEPDV}_{i k, \mu, y}}$ to be solved can be further reduced by Propositions 4(a) and 4(b), respectively. Let $\left(X_{i j k p, y}^{*}, \bar{Z}_{i k p, y}^{*}\right)$ be the optimal solution of $\mathbb{P}_{\mathrm{BKP}_{i k p, \mu, y}}$ with explicit dependence on $y$, and furthermore, let us denote $\mathscr{T}_{r y}$ as $\mathscr{T}_{r y}=\left\{p \in \mathscr{T} \mid \bar{Z}_{i k p, y}^{*} \leq \widetilde{Z}_{i k p, y}^{\max }-r=\widetilde{Z}_{i k p,(y+r)}^{\max }\right\}$ for $\widetilde{Y}_{i k}^{\min } \leq y \leq \widetilde{Y}_{i k}^{\max }$ and $0<r \leq \widetilde{Z}_{i k p, y}^{\max }$. Note that $\mathscr{T}_{r y} \subset$ $\mathscr{T}_{l y} \subset \mathscr{T}$ for $0 \leq l<r \leq \widetilde{Z}_{i k p, y}^{\max }$.

Proposition 4. (a) Given $\left(X_{i j k p, y}^{*}, \bar{Z}_{i k p, y}^{*}\right)$ for some $y \in\left\{y^{\prime} \in\right.$ $\left.\mathbb{Z}^{+} \mid \widetilde{Y}_{i k}^{\min } \leq y^{\prime} \leq \widetilde{Y}_{i k}^{\max }\right\}$ and some $p \in \mathscr{T}$, if $p \in \mathscr{T}_{r y}$ for some $r \in\left\{r^{\prime} \in \mathbb{Z}^{+} \mid 0<r^{\prime} \leq \widetilde{Z}_{i k p, y}^{\max }\right\}$, then $\left(X_{i j k p,(y+r)}^{*}, \bar{Z}_{i k p,(y+r)}^{*}\right)=$ $\left(X_{i j k p, y}^{*}, \bar{Z}_{i k p, y}^{*}\right)$. (b) Given $\left(X_{i j k p, y}^{*}, \bar{Z}_{i k p, y}^{*}\right)$ for some $y \in\left\{y^{\prime} \in \mathbb{Z}^{+} \mid \widetilde{Y}_{i k}^{\min } \leq\right.$ $\left.y^{\prime} \leq \widetilde{Y}_{i k}^{\max }\right\}$ and all $p \in \mathscr{T}$, if $\sum_{p \in \mathscr{T}_{r y}} h_{i k p}^{S} \geq|\mathscr{T}| h_{i k}^{L}$ for some $r \in\left\{r^{\prime} \in \mathbb{Z}^{+} \mid 0<r^{\prime} \leq \widetilde{Z}_{i k p, y}^{\max }\right\}$, then $v_{S E P D V}^{i k}(\mu, y+l) \geq$ $v_{S E P D V}^{i k}(\mu, y+r)$ for $0 \leq l<r$.

In summary, we now present the solution procedure for $\mathbb{P}_{\text {SEPDV }_{i k, \mu}}$ PROC(SEPDV $\left.i k, \mu\right)$, in Algorithm 1 based on the whole discussion above. For reference, Figure 1 illustrates the relationship among all the problems discussed in this section.

3.3. Finding the Lagrange Multipliers. So far, we examined how to address the problem $\mathbb{P}_{\operatorname{SEPDV}_{i k, \mu}}$ for each $(i, k) \in \mathscr{E}_{\mathrm{IN}}$ when the Lagrangian multiplier $\mu$ is given. We now discuss the procedure for finding the Lagrangian multiplier $\mu$. For the purpose, we adapt the subgradient optimization procedure which is one of the most popular methods to find a good set of Lagrange multipliers [31]. Specifically, the Lagrange multipliers $\mu \equiv\left(\mu_{i j p}^{\prime}, \mu_{i j k p}^{\prime \prime}\right)_{\forall i, j, k, p}$ are generated iteratively as

(i) $\mu_{i j p, s+1}^{\prime}=\mu_{i j p, s}^{\prime}+\gamma_{s} G_{i j p, s}^{\prime}$ for all $(i, j) \in \mathbb{Q}_{k}, p \in \mathscr{T}$,

(ii) $\mu_{i j k p, s+1}^{\prime \prime}=\max \left\{0,\left(\mu_{i j k p, s}^{\prime \prime}+\gamma_{s}\right)\right\}+\gamma_{s} G_{i j p, s}^{\prime}$ for all $k \in \mathscr{T}$, $(i, j) \in \mathbb{Q}_{k}, p \in \mathscr{T}$,

where $G_{i j p, s}^{\prime}$ and $G_{i j k p, s}^{\prime \prime}$ are the subgradients for constraints (2b) and (2d), respectively, and $\gamma_{s}$ is the stepsize at iteration $s$.

When it comes to the subgradients $G_{i j p, s}^{\prime}$ and $G_{i j k p, s}^{\prime \prime}$ in the formulas above, they are determined by the solution of $\mathbb{P}_{\mathrm{LR}_{\mu}}$ given $\mu$ as follows: $G_{i j p, s}^{\prime}=1-\sum_{\left\{k \in \mathscr{K} \mid(i, j) \in \mathbb{Q}_{k}\right\}} X_{i j k p}$ for all $(i, j) \in \mathscr{Q}_{k}, p \in \mathscr{T}$, and $G_{i j k p, s}^{\prime \prime}=X_{i j k p}-\bar{X}_{i j k p}$ for all $k \in \mathscr{K},(i, j) \in \mathbb{Q}_{k}, p \in \mathscr{T}$. Moreover, the stepsize $\gamma_{s}$ at iteration $s$ is determined by

$$
\begin{aligned}
& \gamma_{s} \\
& =\frac{\eta\left[\mathrm{UB}-v\left(\mu_{s}\right)\right]}{\sum_{(i, j) \in \mathbb{Q}} \sum_{p \in \mathscr{T}}\left[\left(G_{i j p, s}^{\prime}\right)^{2}+\sum_{\left\{k \in \mathscr{K} \mid(i, j) \in Q_{k}\right\}}\left(G_{i j k p, s}^{\prime \prime}\right)^{2}\right]},
\end{aligned}
$$

where UB represents the best upper bound of $v_{\mathrm{OPT}}$ found up to iteration $s$ and $\eta$ is a control parameter for $\gamma_{s}$. In this paper, $\eta$ is set to 2 at the beginning and then halved if the lower bound is not improved in a predetermined number of consecutive iterations. 


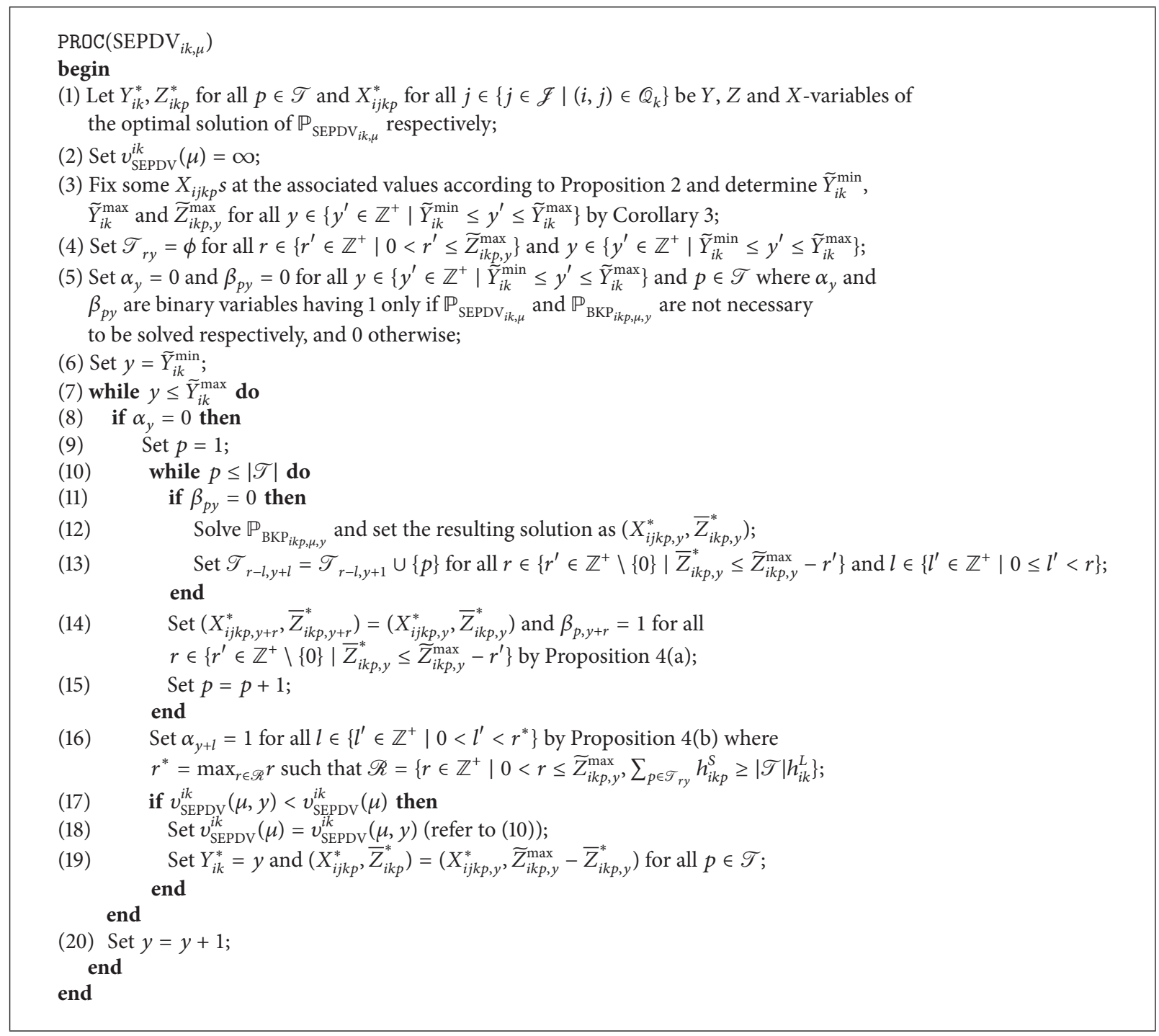

Algorithm 1: Solution procedure for $\mathbb{P}_{\mathrm{SEPDV}_{i k, \mu}}$.

3.4. Finding a Feasible Solution. A solution obtained by solving $\mathbb{P}_{L^{\prime}}$ based on the discussion in Section 3.2 may be infeasible to $\mathbb{P}_{\text {MVLPUC }_{+}}$, and hence we propose a two-phase heuristic to deal with infeasibility issues as follows: In the first phase, a construction heuristic $(\mathrm{CH})$ is derived to modify any infeasible solutions to $\mathbb{P}_{\text {MVLPUC }}$ into feasible ones, and then, in the second phase, a tabu search-based heuristic (TSH) is utilized to improve the solution of $(\mathrm{CH})$. The details are presented in the subsequent sections.

3.4.1. Construction Heuristic (CH). As mentioned above, a construction heuristic $(\mathrm{CH})$ intends to deal with possible infeasibility issues of solutions obtained by solving $\mathbb{P}_{\mathrm{LR}_{\mu}}$, and $(\mathrm{CH})$ is performed at each subgradient iteration. Let $X_{i j k p}^{*}$ and $\bar{X}_{i j k p}^{*}$ be the $X$ - and $\bar{X}$-variable values of the optimal solution of $\mathbb{P}_{\mathrm{SEPDV}_{i k, \mu}}$ and $\mathbb{P}_{\mathrm{SEPDV}_{k j, \mu}}$, respectively. First, the corresponding demand for all $(i, j) \in \mathbb{Q}$ and $p \in \mathscr{T}$ is reassigned to the UFCC with the lexicographical maximum value of the three-tuple relation $\left(\left(X_{i j k p}^{*}+\bar{X}_{i j k p}^{*}\right),\left(\theta_{i j k p} X_{i j k p}^{*}+\right.\right.$ $\left.\left.\delta_{i j k p} \bar{X}_{i j k p}^{*}\right),-\left(h_{i k}^{L}+h_{k j}^{L}\right)\right)$. Then, from the reassigned demands, demand quantity $D_{e p}$ for all $e \in \mathscr{E}$ and $p \in \mathscr{T}$ is computed as $D_{e p}=\sum_{\left\{j \in \mathscr{F} \mid(i, j) \in \mathbb{Q}_{k}\right\}} d_{i j p} X_{i j k p}$ or $D_{e p}=\sum_{\left\{i \in \mathscr{I} \mid(i, j) \in \mathbb{Q}_{k}\right\}} d_{i j p} \bar{X}_{i j k p}$. Finally, to allocate vehicles for each $e \in \mathscr{E}$ at the minimum vehicle cost, we solve the following vehicle allocation problem (VAP), $\mathbb{P}_{\mathrm{VAP}_{e}}$.

Problem $\mathbb{P}_{V A P_{e}}$

$$
\begin{array}{ll}
v_{\mathrm{VAP}}^{e}=\min & |\mathscr{T}| h_{e}^{L} Y_{e}+\sum_{p \in \mathscr{T}} h_{e p}^{S} Z_{e p} \\
\text { subject to } & D_{e p} \leq \Gamma\left(Y_{e}+Z_{e p}\right), \quad \forall p \in \mathscr{T} \\
& Y_{e}, Z_{e p} \in \mathbb{Z}^{+}, \quad \forall p \in \mathscr{T} .
\end{array}
$$

Proposition 5 characterizes the optimal solutions of $\mathbb{P}_{\mathrm{VAP}_{e}}$. 


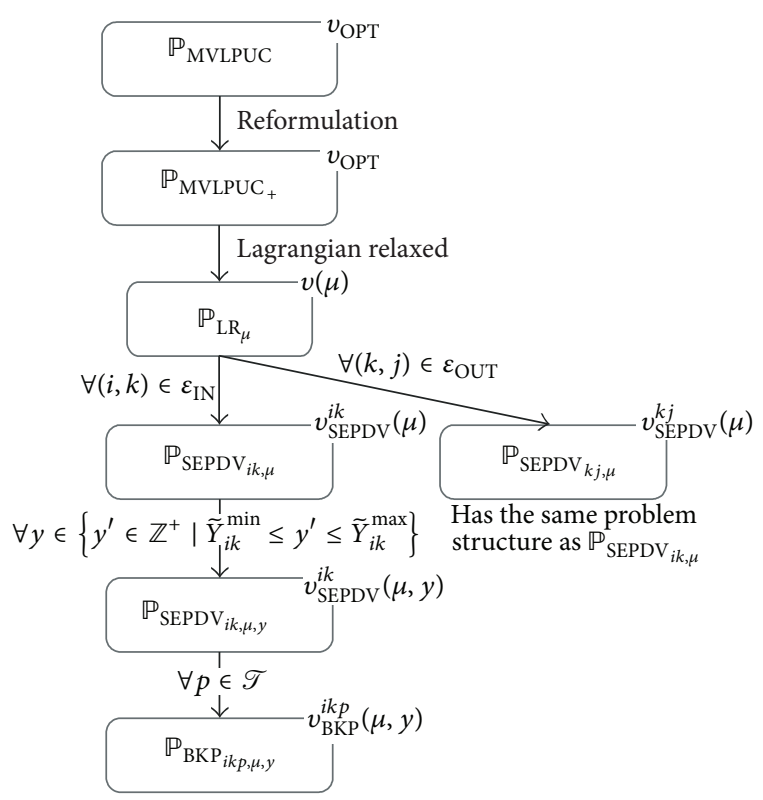

Figure 1: Problem decomposition of $\mathbb{P}_{\text {MVLPUC }}$.

Proposition 5. There is an optimal solution of $\mathbb{P}_{V A P_{e}}$ with $\left(Y_{e}, Z_{e p}\right) \in\left\{\left(y, Z_{e p, y}\right) \mid y=y_{[1]}, \ldots, y_{[|\mathscr{T}|]}\right\}$, where $Z_{e p, y}=$ $\max \left\{0,\left(\left\lceil D_{e p} / \Gamma\right\rceil-y\right)\right\}$ and $y_{[i]}$ is the ith largest $y$.

Proof. As in Corollary 3, it is straightforward to show that there is an optimal solution of $\mathbb{P}_{\mathrm{VAP}_{e}}$ with $\left(Y_{e}, Z_{e p}\right) \in$ $\left\{\left(y, Z_{e p, y}\right) \mid y_{[1]} \leq y \leq y_{[|\mathscr{T}|]}\right\}$. For an integer $m$ such that $y_{[n]} \leq m<y_{[n+1]}$ and for $n \in\{1, \ldots,|\mathscr{T}|-1\}$,

$$
\begin{aligned}
Z_{e[p], m} & = \begin{cases}0 & \text { if } p \leq n \\
\left(y_{[p]}-m\right) & \text { otherwise }\end{cases} \\
Z_{e[p], m+1} & = \begin{cases}0 & \text { if } p \leq n \\
\left(y_{[p]}-(m+1)\right) & \text { otherwise. }\end{cases}
\end{aligned}
$$

Let $v_{\mathrm{VAP}}^{e}(y)$ be the value of the objective function (12a) associated with $Y_{e}$ fixed at $y \in\left[y_{1}, y_{[|\mathscr{T}|]}\right]$ in $\mathbb{P}_{\mathrm{VAP}_{e}}$. Then, $v_{\mathrm{VAP}}^{e}(m+1)-v_{\mathrm{VAP}}^{e}(m)=\left[(m+1)|\mathscr{T}| h_{e}^{L}-\sum_{p=1}^{|\mathscr{T}|} Z_{e[p], m+1}\right]-$ $\left[m|\mathscr{T}| h_{e}^{L}-\sum_{p=1}^{|\mathscr{T}|} Z_{e[p], m}\right]=|\mathscr{T}| h_{e}^{L}-\sum_{p=n+1}^{|\mathscr{T}|} h_{e[p]}^{S}$. It implies that $v_{\mathrm{VAP}}^{e}(m)$ is a linear function in $m$ over the range $y_{[n]} \leq$ $m<y_{[n+1]}$. Therefore, there is an optimal solution of $\mathbb{P}_{\mathrm{VAP}_{e}}$ with $\left(Y_{e}, Z_{e p}\right) \in\left\{\left(y, Z_{e p, y}\right) \mid y=y_{[1]}, \ldots, y_{[|\mathscr{T}|]}\right\}$.

By Proposition 5, $\mathbb{P}_{\mathrm{VAP}}$ can be solved by finding a solution of having the minimum cost among $|\mathscr{T}|$ number of solutions which are associated with $Y_{e}$ fixed at $y_{[p]}$ for all $p \in \mathscr{T}$.

3.4.2. Tabu Search-Based Heuristic (TSH). After the first phase $(\mathrm{CH})$, a tabu search-based heuristic (TSH) is performed to improve the solution obtained from $(\mathrm{CH})$. We note that (TSH) is expected to require much more computational time than $(\mathrm{CH})$, and hence, to save the elapsed time of the overall algorithm, (TSH) is executed only at the subgradient iterations satisfying the following two conditions ((a) and (b)) together: (a) the best lower bound is improved and (b) the solution obtained by $(\mathrm{CH})$ at the current subgradient iteration is different from the solutions obtained by $(\mathrm{CH})$ so far. In this paper, we call the conditions (a) and (b) as "(TSH)-activating conditions."

Tabu search is one of the popular metaheuristics designed to escape the trap of local optimality [32], and it has been used frequently in various network-related problems as in Sung and Song [25], Crevier et al. [33], and Üster and Maheshwari [34]. We now explain the main features of the tabu search used in (TSH), including move operation, tabu restriction and aspiration criterion, move evaluation, and termination condition.

The move operation used in this paper is to modify assignment of some freight demand, say $q=(i, j) \in \mathbb{Q}$, from its current UFCC, say $l \in \mathscr{K}$, to the alternative UFCC, say $k \in$ $\mathscr{K}$, for some period, say $p \in \mathscr{T}$. It corresponds to modifying the value of $X_{i j l p}$ from 1 to 0 and the value of $X_{i j k p}$ from 0 to 1 in Problem $\mathbb{P}_{\text {MVLPUC }}$. The tabu restriction is employed such that the UFCC assignment for any freight demand should not be changed during tabu_size number of iterations except for the case that satisfies the aspiration criterion (to be explained later). To implement the tabu restriction, as a new solution is selected, the information of the freight demand associated with the move operation is recorded in a list (called tabu list) and kept in the list over the tabu_size number of iterations. In this paper, the tabu_size is chosen randomly from the discrete uniform distribution in the closed interval $[10,20]$. The aspiration criterion adapted here is as follows: a solution from the move operation for the freight demands recorded in the tabu list is eligible to be chosen as a new solution only if the move improves the best upper bound. Moreover, when we choose a new solution among neighbor solutions at each iteration, the move evaluation function used in this paper is to maximize $\{\mathrm{mv}-A \cdot n\}$ where $\mathrm{mv}, n$, and $A$ represent the decrease amount of the objective function value, the frequency that the corresponding move is used to produce a new solution so far, and the associated penalty with the frequency, respectively. When it comes to $\mathrm{mv}$, it is computed simply by subtracting the objective value of the new solution from the objective value of the current solution, and if $\mathrm{mv}$ is positive, $A$ is set to 0 and 50 otherwise. We note that this move evaluation function is known to have an advantage in diversifying the search direction efficiently [32]. Lastly, if the total number of performed iterations amounts to max_iter or the best upper bound has not been improved during max_no_imp consecutive iterations, (TSH) is terminated. In this paper, max_iter and max_no_imp are set at 500 and 30, respectively.

3.5. Overall Solution Algorithm. We now summarize all the discussion and present the algorithm PROC(MVLPUC) for solving $\mathbb{P}_{\text {MVLPUC }}$ in Algorithm 2. It can be terminated either after a predetermined number (1000 in this paper) of iterations or when $\eta$ gets too small (i.e., $\eta \leq 0.005$ in this paper). It can also be terminated when $(((\mathrm{UB}-\mathrm{LB}) / \mathrm{LB}) \times 100)$ 


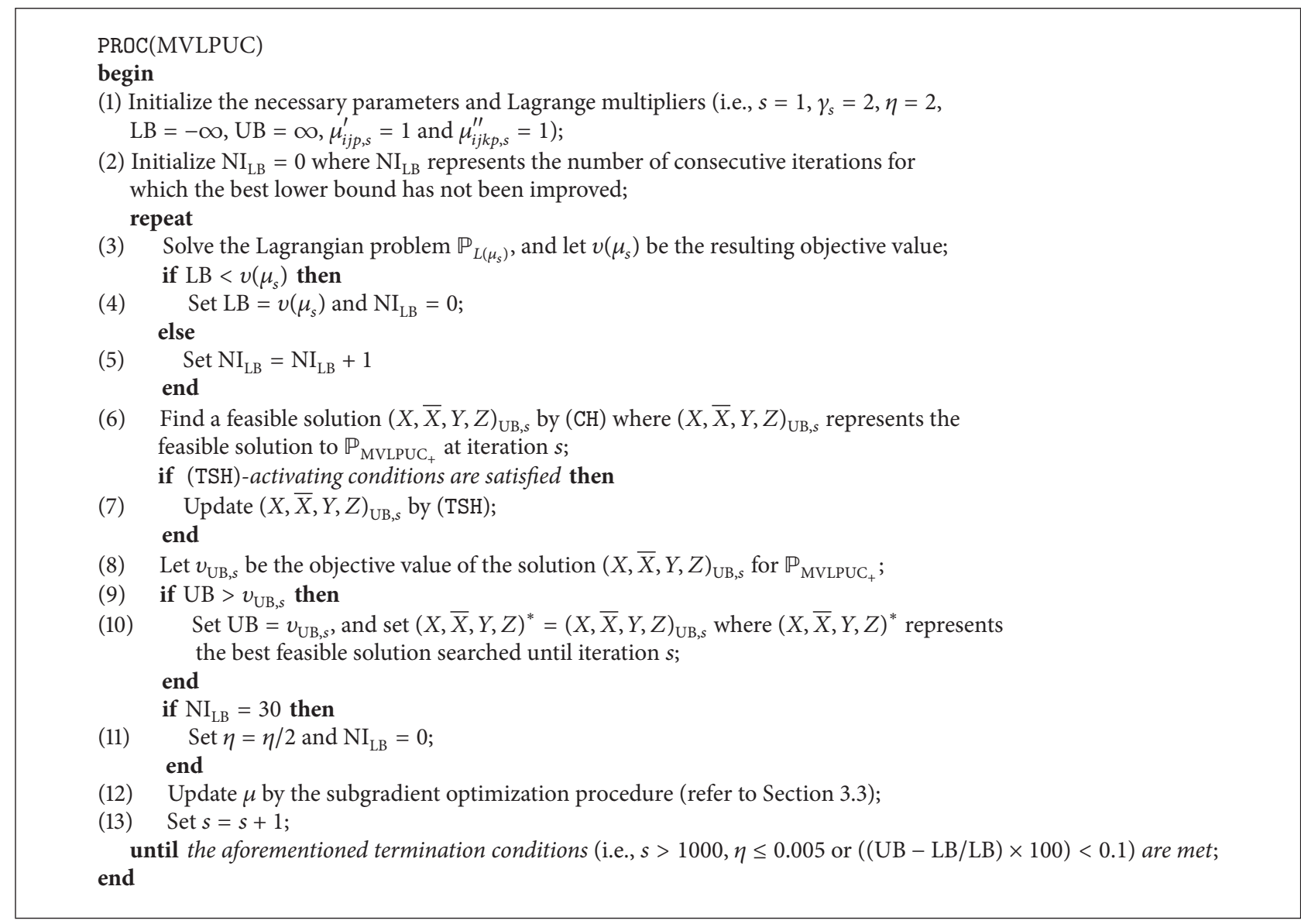

Algorithm 2: Overall solution procedure for $\mathbb{P}_{\text {MVLPUC }}$.

is less than $0.1 \%$, where UB and LB represent the best upper and lower bounds searched so far, respectively.

\section{Numerical Experiments}

This section presents the numerical results on the performance of the proposed Lagrangian heuristic algorithm PROC(MVLPUC) for $\mathbb{P}_{\text {MVLPUC. The algorithm PROC(MVLPUC) }}$ was implemented in $\mathrm{C} / \mathrm{C}++$ language, and the performance of PROC(MVLPUC) is compared with those of the following three algorithms as benchmarks: (a) the Lagrangian heuristic where only $(\mathrm{CH})$ is used in PROC(MVLPUC) while (TSH) is excluded, (b) a commercial optimization software IBM ILOG CPLEX Optimization Studio, and (c) the algorithm where $(\mathrm{CH})$ is applied at every node of the branch-and-bound tree for IBM ILOG CPLEX. The three algorithms are denoted by $(\mathrm{LCH}),(\mathrm{CX})$, and $(\mathrm{CXCH})$, respectively.

For the numerical experiments, a variety of problem instances were randomly generated according to the underlying network structure, the number of nodes (i.e., $|\mathscr{V}|$ ), and the number of time periods (i.e., $|\mathscr{T}|$ ). Three types of underlying networks were considered in the experiments including origin-centric, equal, and destination-centric networks according to the number of origin and destination nodes. Origin-centric networks (destination-centric networks) have larger numbers of origin (destination) nodes than those of destination (origin) nodes, and equal networks have almost equivalent numbers of origin nodes as those of destination nodes. Moreover, three $|\mathscr{V}|$ values including 30, 40, and 50 and three $|\mathscr{T}|$ including 3,6 , and 12 were considered in the experiment.

To generate problem instances, each node was randomly selected on a $100 \times 100$ grid. Some of the nodes were connected by arcs, which were also chosen randomly. Unitperiod long-term vehicle lease cost $h_{e}^{L}$ was calculated based on Euclidean distance between nodes, and unit-period shortterm vehicle lease cost $h_{e p}^{S}$ was randomly chosen in the range from 1.5 to 2 times of $h_{e}^{L}$. Such a structure of vehicle lease costs reflects the actual practice in Korean logistics industry. Moreover, transportation time $t_{e}$ was randomly chosen in the range from 0.8 to 1.2 times of $h_{e}^{L}$, and handling time at $k \in \mathscr{K}$, $o_{k}$, was set as $o_{k}=\min \left\{\min _{i \in \mathcal{F}} t_{i k}, \min _{j \in \mathcal{F}} t_{k j}\right\}$. Transportation time restriction $\mathrm{TL}_{i j}$ was set as $\mathrm{TL}_{i j}=0.1\left(\min _{k \in \mathscr{K}}\left\{t_{i k}+o_{k}+\right.\right.$ $\left.\left.t_{k j}\right\}\right)+0.9\left(\max _{k \in \mathscr{K}}\left\{t_{i k}+o_{k}+t_{k j}\right\}\right)$. Demand quantity $d_{i j p}$ was randomly selected in the range from 1 to 90 , and vehicle capacity $\Gamma$ was set at 100 . For each combination of underlying network structure, number of nodes, and number of periods, four problem instances were generated, and hence, in total, 108 problem instances were generated. 
Tables 1-3 show the experimental results for the problem instances with $|\mathscr{V}|=30,40$ and 50 nodes, respectively. The tables present the best lower bound (LB), the best upper bound (UB), the percentage deviation between the two (\%Gap), and the elapsed time in seconds (Time $[\mathrm{s}]$ ). The percentage deviation, $\% \mathrm{Gap}$, is computed as $((\mathrm{UB}-\mathrm{LB}) / \mathrm{LB}) \times$ 100.

For the problem instances with $|\mathscr{V}|=30$, PROC(MVLPUC) gave solutions with the following performance: (a) the duality gap (shown in the "\%Gap" column) ranges from $5.26 \%$ to $12.99 \%$ with the average of $7.98 \%$, and (b) the elapsed time ranges from $33.77 \mathrm{~s}$ to $691.83 \mathrm{~s}$ with the average of $175.12 \mathrm{~s}$. The duality gap does not seem to be dependent on both $|\mathscr{T}|$ and the network structure, as observed that the duality gap does not change consistently according to them. On the other hand, the elapsed time seems to increase not exponentially but linearly as $|\mathscr{T}|$ increases while it does not seem to be dependent on the network structure. For example, the average elapsed times for the problem instances with $|\mathscr{T}|=3,6$, and 12 are $80.06 \mathrm{~s}$, $158.05 \mathrm{~s}$, and $287.26 \mathrm{~s}$, respectively. The aforementioned trends can be observed in the problem instances with $|\mathscr{V}|=40$ and 50 as well.

From all the experiments, the performance changes of the proposed algorithm according to the number of nodes (i.e., $|\mathscr{V}|)$ can be summarized as follows. As $|\mathscr{V}|$ increases, the duality gap seems to marginally increase. For example, the average duality gaps for the problem instances with $|\mathscr{V}|=$ 30,40 , and 50 are $7.98 \%, 8.58 \%$, and $9.44 \%$, respectively, and this may be due to the fact that, as $|\mathscr{V}|$ increases, the number of the associated Lagrange multipliers greatly increases as shown in $\mu_{i j k p}^{\prime \prime}$, and hence, the associated lower bound may become looser accordingly. We also observe that, as $|\mathscr{V}|$ increases, the elapsed time increases rapidly, but not exponentially. For example, the average elapsed times for the problem instances with $|\mathscr{V}|=30,40$, and 50 are $175.12 \mathrm{~s}$, $654.59 \mathrm{~s}$, and $2591.26 \mathrm{~s}$, respectively.

We now compare the performance of PROC(MVLPUC) with those of the aforementioned three algorithms including $(\mathrm{LCH}),(\mathrm{CX})$, and $(\mathrm{CXCH})$, respectively. They were tested on the small-sized problem instances (or instances with $|\mathscr{V}|=30)$. For $(\mathrm{LCH})$, the same termination conditions as in PROC(MVLPUC) were used, while for (CX) and ( $\mathrm{CXCH})$, an execution time limit of five hours was applied as a termination condition.

Table 4 shows the numerical results of the compared algorithms for the problem instances with $|\mathscr{V}|=30$. The table gives the percentage deviations between the best lower bounds obtained from each of the compared algorithms and the best lower bound obtained by the proposed algorithm (LB in Table 1) and the percentage deviations between the best upper bounds obtained by each of the compared algorithms and the best upper bound obtained by the proposed algorithm (UB in Table 1). For (LCH) and both of (CX) and $(\mathrm{CXCH})$, the percentage deviations of the lower bounds are denoted by $\mathrm{ILB}_{\mathrm{LCH}}$ and $\mathrm{ILB}_{\mathrm{CX}}$, respectively. Note that (CX) and $(\mathrm{CXCH})$ give the same lower bound obtained from the

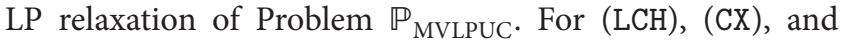

$(\mathrm{CXCH})$, the percentage deviations of the upper bounds are denoted by $\mathrm{IUB}_{\mathrm{LCH}}, \mathrm{IUB}_{\mathrm{CX}}$, and $\mathrm{IUB}_{\mathrm{CXCH}}$, respectively. In detail, the percentage deviations of the lower bounds and the percentage deviations of the upper bounds were computed by $\left(\left(\mathrm{LB}-\mathrm{LB}_{\mathrm{C}}\right) / \mathrm{LB}_{C}\right) \times 100$ and $\left(\left(\mathrm{UB}_{C}-\mathrm{UB}\right) / \mathrm{UB}_{C}\right) \times 100$, respectively, where $\mathrm{LB}_{C}$ and $\mathrm{UB}_{C}$ are the best lower bound and the best upper bound obtained by the corresponding compared algorithm, respectively. For example, if $\mathrm{IUB}_{\mathrm{LCH}}$ is positive, it means that the best upper bound of the proposed algorithm is better than that of (LCH) at the associated rate. From the table, the proposed algorithm may give the almost same lower bounds as (LCH) and better upper bounds than $(\mathrm{LCH})$ from the fact that $\mathrm{ILB}_{\mathrm{LCH}}$ is almost zero and $\mathrm{ULB}_{\mathrm{LCH}}$ ranges from $0.36 \%$ to $11.88 \%$. This may imply that (TSH) embedded in PROC(MVLPUC) improves the upper bound significantly. Moreover, PROC(MVLPUC) may give better lower bounds than $(\mathrm{CX})$ and $(\mathrm{CXCH})$. For the long planning horizon, PROC(MVLPUC) may give better upper bounds than $(\mathrm{CX})$ and $(\mathrm{CXCH})$, while for the short planning horizon, it may not. For example, the associated average values of IUB $_{\mathrm{CX}}$ with $|\mathscr{T}|=3,6$, and 12 are $-2.35 \%, 2.56 \%$, and $6.44 \%$, respectively. This may imply that the proposed algorithm is suitable for the large-sized problem instances. Also, it can be observed that (CXCH) outperforms (CX) marginally.

In summary, the proposed algorithm PROC(MVLPUC) may give good lower and upper bounds within reasonable time, and the duality gap seems not to be dependent on the network structure and the number of time periods, while it seems to increase a little, as $|\mathscr{V}|$ increases. Furthermore, as presented in Table 3, the average duality gap for the problem instances with $|\mathscr{V}|=50$ is still less than $10 \%$, and the elapsed time seems not to be dependent on the network structure, while it increases as $|\mathscr{V}|$ or $|\mathscr{T}|$ increases. However, the increase in the elapsed time is not exponential in either case.

\section{Concluding Remarks}

In this paper, a multiperiod vehicle lease planning problem in an urban freight distribution network with UFCCs is considered where vehicles for each direct service are leased in short term or long term. The problem under consideration is motivated by the realistic situation where the freight demands fluctuate dynamically in time, and urban freight distribution can be made through UFCCs. The problem is formulated as an integer programming model and can be proven to be NP-hard in a strong sense. Thus, we propose a Lagrangian-based heuristic to address the concomitant problem. Various numerical experiments are performed to evaluate the performance of the proposed algorithm, and they indicate that the proposed algorithm works well. The proposed algorithm may be applied to vehicle lease planning for industrial logistic services for handling various products including food, apparel, electronic goods, and logistics companies.

As extended research, several models derived from the proposed model in this paper can be considered. For example, a problem where each freight demand can be delivered through more than a single UFCC may be interesting. 


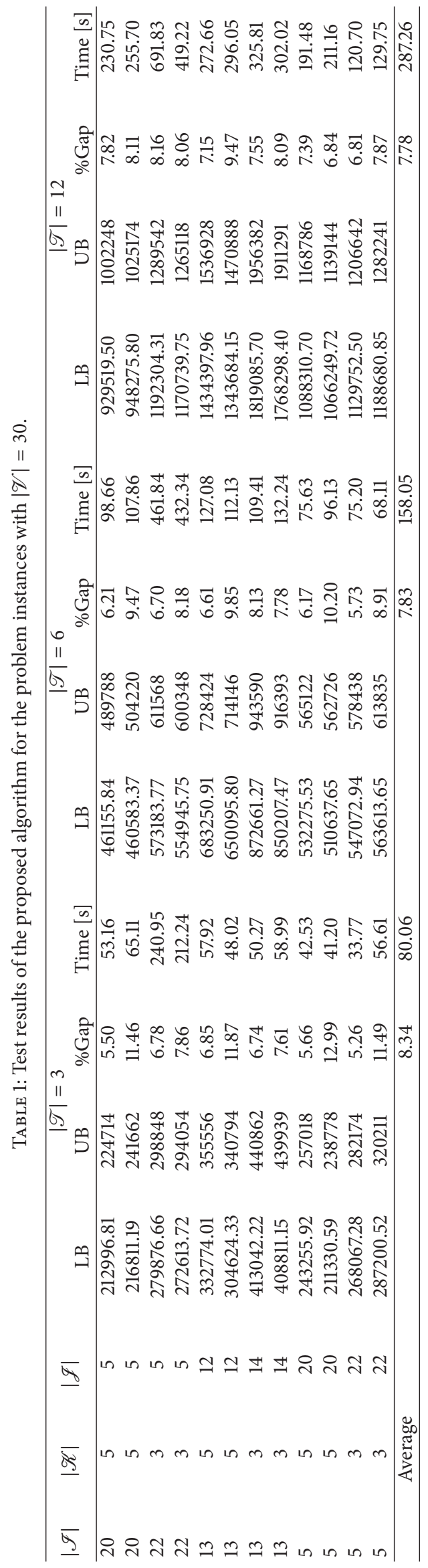




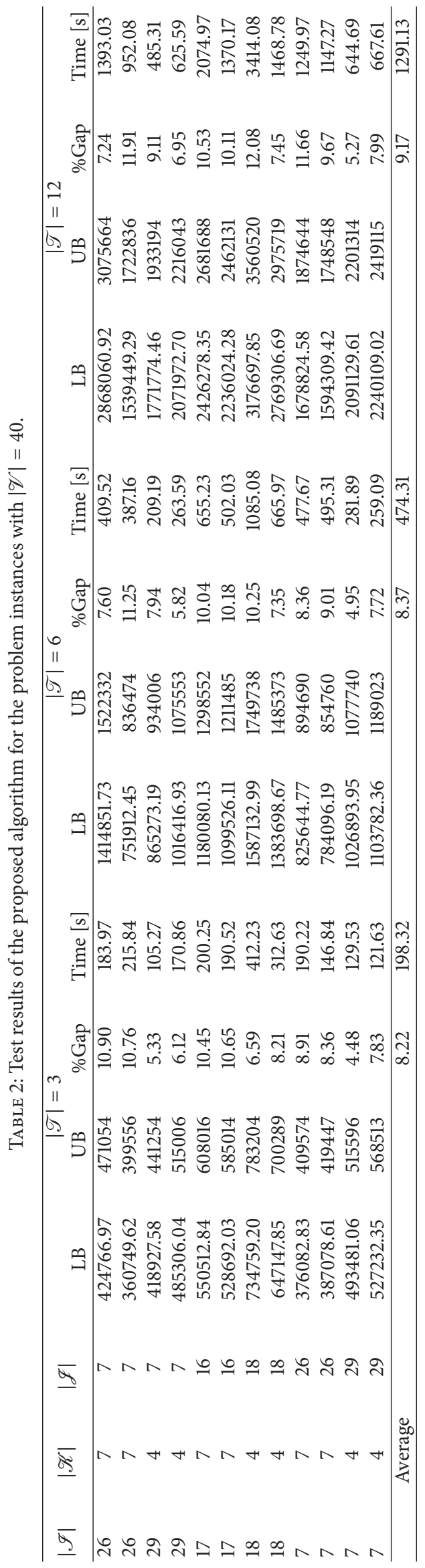




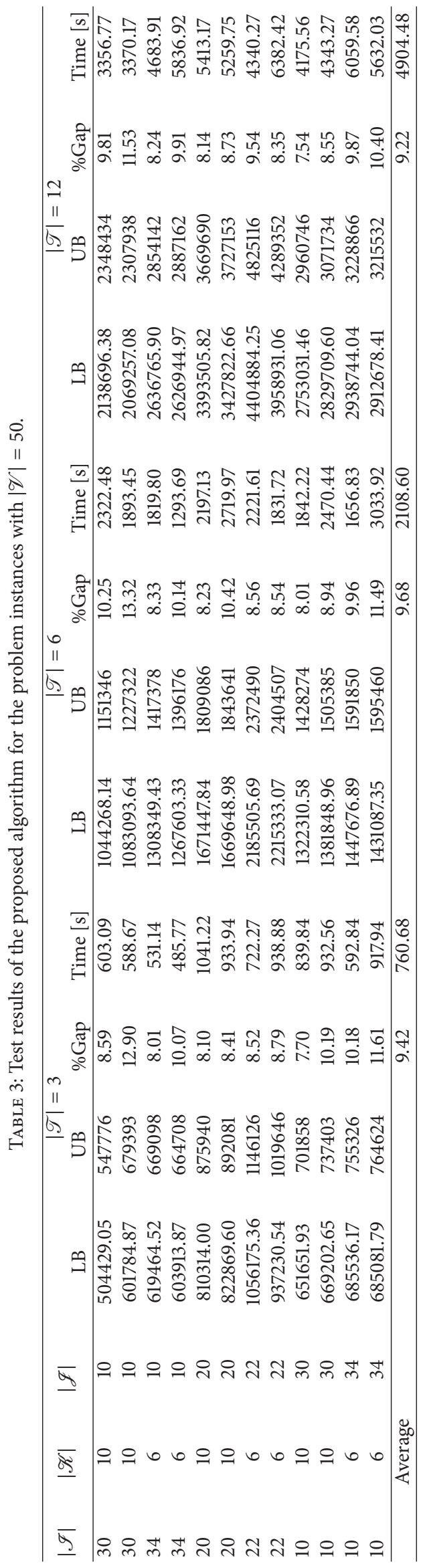


TABLE 4: Test results of the compared algorithms for the problem instances with $|\mathscr{V}|=30$.

\begin{tabular}{|c|c|c|c|c|c|c|c|c|c|c|c|c|c|c|c|c|c|}
\hline \multirow{2}{*}{\multicolumn{2}{|c|}{$|\mathscr{I}||\mathscr{K}|$}} & \multirow{2}{*}{$|\mathscr{F}|$} & \multicolumn{5}{|c|}{$|\mathscr{T}|=3$} & \multicolumn{5}{|c|}{$|\mathscr{T}|=6$} & \multicolumn{5}{|c|}{$|\mathscr{T}|=12$} \\
\hline & & & $\mathrm{ILB}_{\mathrm{LCH}}$ & ILB $_{C X}$ & $\mathrm{IUB}_{\mathrm{LCH}}$ & $\mathrm{IUB}_{\mathrm{CX}}$ & $\mathrm{IUB}_{\mathrm{CXCH}}$ & $\mathrm{ILB}_{\mathrm{LCH}}$ & ILB $_{C X}$ & $\mathrm{IUB}_{\mathrm{LCH}}$ & $\mathrm{IUB}_{\mathrm{CX}}$ & $\mathrm{IUB}_{\mathrm{CXCH}}$ & $\mathrm{ILB}_{\mathrm{LCH}}$ & ILB $_{C X}$ & $\mathrm{IUB}_{\mathrm{LCH}}$ & $\mathrm{IUB}_{\mathrm{CX}}$ & $\mathrm{IUB}_{\mathrm{CXCH}}$ \\
\hline 20 & 5 & 5 & 0.01 & 10.58 & 5.52 & -0.17 & -1.17 & -0.19 & 11.45 & 3.73 & 1.38 & 1.16 & -0.09 & 9.37 & 3.53 & 4.14 & 0.26 \\
\hline 20 & 5 & 5 & 0.00 & 11.14 & 3.90 & -6.46 & -6.35 & -0.14 & 10.38 & 4.68 & -2.77 & -2.27 & -0.08 & 10.21 & 4.66 & 3.61 & -0.32 \\
\hline 22 & 3 & 5 & 0.45 & 9.55 & 11.88 & 2.30 & -0.43 & 0.00 & 9.18 & 8.11 & 10.27 & 2.05 & 0.09 & 9.22 & 4.08 & 7.54 & 4.11 \\
\hline 22 & 3 & 5 & -0.01 & 8.48 & 4.00 & -3.38 & -0.76 & 0.02 & 8.88 & 6.29 & -0.47 & -1.72 & 0.04 & 8.65 & 3.97 & 9.44 & 4.98 \\
\hline 13 & 5 & 12 & -0.14 & 10.05 & 2.80 & 1.12 & -1.10 & -0.13 & 9.25 & 7.05 & 3.18 & 1.5 & -0.71 & 8.34 & 3.92 & 6.77 & 38 \\
\hline 13 & 5 & 12 & -0.31 & 9.50 & 5.78 & -2.84 & -2.72 & -0.56 & 8.76 & 6.76 & 1.07 & 1.87 & 0.11 & 9.65 & 6.44 & 7.89 & 6.85 \\
\hline 13 & 3 & 14 & -0.26 & 5.17 & 5.96 & 1.13 & 0.01 & -0.17 & 5.52 & 4.07 & 8.08 & 313 & -0.09 & 5.60 & 5.75 & 11.74 & 7.45 \\
\hline 13 & 3 & 14 & -0.20 & 6.19 & 5.09 & -1.86 & -1.45 & -0.07 & 6.33 & 5.61 & 8.67 & 5.70 & -0.08 & 6.29 & 6.29 & 9.87 & 8.04 \\
\hline 5 & 5 & 20 & -0.05 & 15.65 & 4.44 & -1.48 & -1.26 & -0.56 & 16.84 & 5.09 & 1.00 & 0.12 & -0.26 & 16.59 & 3.19 & 0.36 & 1.32 \\
\hline J & 5 & 20 & -0.02 & 12.99 & 0.36 & -8.46 & -8.34 & 0.00 & 16.94 & 3.87 & -5.58 & -5.31 & -0.16 & 16.57 & 4.13 & 1.42 & 0.17 \\
\hline 5 & 3 & 22 & -0.06 & 13.93 & 2.91 & -1.57 & -1.87 & 0.02 & 13.92 & 3.82 & 0.99 & -0.39 & -0.58 & 12.42 & 3.86 & 4.14 & 6.70 \\
\hline 5 & 3 & 22 & -0.01 & 13.45 & 3.48 & -6.51 & -6.97 & -0.16 & 12.66 & 3.17 & -2.62 & -2.75 & -0.68 & 11.69 & 2.96 & 5.66 & 3.84 \\
\hline \multicolumn{3}{|c|}{ dverage } & -0.05 & 10.56 & 4.68 & -2.35 & -2.70 & -0.18 & 10.39 & 5.13 & 2.56 & 0.69 & -0.16 & 9.97 & 4.47 & 6.44 & 4.32 \\
\hline
\end{tabular}

Moreover, it may be necessary to deal with vehicle specific lease periods. Lastly, proposing the efficient heuristic-based approach for solving large-scale problems would be the topic for the future research.

\section{Conflict of Interests}

The authors declare that there is no conflict of interests regarding the publication of this paper.

\section{Acknowledgment}

Sang Hwa Song's work was supported by Industrial Strategic Technology Development Program (Grant no. 10044535), funded by the Ministry of Trade, Industry and Energy.

\section{References}

[1] J. F. Ehmke, "City logistics," in Integration of Information and Optimization Models for Routing in City Logistics, chapter 2, Springer New York, 2012.

[2] E. Taniguchia, R. G. Thompsonb, and T. Yamada, "Recent trends and innovations in modelling city logistics," Procedia-Social and Behavioral Sciences, vol. 125, pp. 4-14, 2014.

[3] E. E. Blanco and J. C. Fransoo, "Reaching 50 million nanostores: retail distribution in emerging megacities," TUE Working Paper 404, 2013.

[4] N. Anand, H. Quak, R. van Duin, and L. Tavasszy, "City logistics modeling efforts: trends and gaps-a review," Procedia-Social and Behavioral Sciences, vol. 39, pp. 101-115, 2012.

[5] T. G. Crainic, N. Ricciardi, and G. Storchi, "Models for evaluating and planning city logistics systems," Transportation Science, vol. 43, no. 4, pp. 432-454, 2009.

[6] J. F. Ehmke, A. Steinert, and D. C. Mattfeld, "Advanced routing for city logistics service providers based on time-dependent travel times," Journal of Computational Science, vol. 3, no. 4, pp. 193-205, 2012.
[7] J. Yang, J. Guo, and S. Ma, "Low-carbon city logistics distribution network design with resource deployment," Journal of Cleaner Production, 2013.

[8] E. Marcucci and R. Danielis, "The potential demand for a urban freight consolidation centre," Transportation, vol. 35, no. 2, pp. 269-284, 2008.

[9] M. Browne, J. Allen, and J. Leonardi, "Evaluating the use of an urban consolidation centre and electric vehicles in central London," IATSS Research, vol. 35, no. 1, pp. 1-6, 2011.

[10] L. Dablanc, G. Giuliano, K. Holliday, and T. O’Brien, "Best practices in urban freight management: lessons from an international survey," Transportation Research Record, no. 2379, pp. 29-38, 2013.

[11] C. Macharis and S. Melo, City Distribution and Urban Freight Transport: Multiple Perspectives, Edward Elgar Publishing, Cheltenham, UK, 2011.

[12] F. Su and M. J. Roorda, "The potential of urban freight consolidation for the Toronto central business district," in Proceedings of the 93rd Annual Meeting of the Transportation Research Board, Washington, DC, USA, January 2014.

[13] M. K. Triantafyllou, T. J. Cherrett, and M. Browne, "Urban freight consolidation centers: a case study in the UK retail sector," in Proceedings of the Transportation Research Board 93rd Annual Meeting, 2014.

[14] Y. Zhou and X. Wang, "Decision-making process for developing urban freight consolidation centers: analysis with experimental economics," Journal of Transportation Engineering, vol. 140, no. 2, 2014.

[15] J. F. Ehmke, S. Meisel, and D. C. Mattfeld, "Floating car based travel times for city logistics," Transportation Research Part C: Emerging Technologies, vol. 21, no. 1, pp. 338-352, 2012.

[16] M. A. Figliozzi, "Analysis of the efficiency of urban commercial vehicle tours: data collection, methodology, and policy implications," Transportation Research Part B: Methodological, vol. 41, no. 9, pp. 1014-1032, 2007.

[17] J.-B. Sheu, "A novel dynamic resource allocation model for demand-responsive city logistics distribution operations," Transportation Research Part E: Logistics and Transportation Review, vol. 42, no. 6, pp. 445-472, 2006. 
[18] K. R. Gue, "Effects of trailer scheduling on the layout of freight terminals," Transportation Science, vol. 33, no. 4, pp. 419-428, 1999.

[19] J. J. Bartholdi III and K. R. Gue, "Reducing labor costs in an LTL crossdocking terminal," Operations Research, vol. 48, no. 6, pp. 823-832, 2000.

[20] J. J. Bartholdi III and K. R. Gue, “The best shape for a crossdock," Transportation Science, vol. 38, no. 2, pp. 235-244, 2004.

[21] Y. Li, A. Lim, and B. Rodrigues, "Crossdocking-JIT scheduling with time windows," Journal of the Operational Research Society, vol. 55, no. 12, pp. 1342-1351, 2004.

[22] H. D. Ratliff, J. V. Vate, and M. Zhang, "Network design for loaddriven cross-docking systems," GIT Technical Report, 1999.

[23] P. Chen, Y. Guo, A. Lim, and B. Rodrigues, "Multiple crossdocks with inventory and time windows," Computers and Operations Research, vol. 33, no. 1, pp. 43-63, 2006.

[24] H. Donaldson, E. L. Johnson, H. D. Ratliff, and M. Zhang, "Schedule-driven cross-docking networks," Tech. Rep., GIT, 1999.

[25] C. S. Sung and S. H. Song, "Integrated service network design for a cross-docking supply chain network," Journal of the Operational Research Society, vol. 54, no. 12, pp. 1283-1295, 2003.

[26] C. S. Sung and W. Yang, "An exact algorithm for a crossdocking supply chain network design problem," Journal of the Operational Research Society, vol. 59, no. 1, pp. 119-136, 2008.

[27] D. A. Serel, M. Dada, and H. Moskowitz, "Sourcing decisions with capacity reservation contracts," European Journal of Operational Research, vol. 131, no. 3, pp. 635-648, 2001.

[28] R. W. Seifert, U. W. Thonemann, and W. H. Hausman, "Optimal procurement strategies for online spot markets," European Journal of Operational Research, vol. 152, no. 3, pp. 781-799, 2004.

[29] L. van Norden and S. van de Velde, "Multi-product lot-sizing with a transportation capacity reservation contract," European Journal of Operational Research, vol. 165, no. 1, pp. 127-138, 2005.

[30] P. Toth, "Dynamic programming algorithms for the Zero-One Knapsack problem," Computing, vol. 25, no. 1, pp. 29-45, 1980.

[31] C. R. Reeves, Modern Heuristic Techniques for Combinatorial Problems, John Wiley \& Sons, 1993.

[32] F. Glover and M. Laguna, Tabu Search, Kluwer Academic Publishers, Boston, Mass, USA, 1997.

[33] B. Crevier, J.-F. Cordeau, and G. Laporte, "The multi-depot vehicle routing problem with inter-depot routes," European Journal of Operational Research, vol. 176, no. 2, pp. 756-773, 2007.

[34] H. Üster and N. Maheshwari, "Strategic network design for multi-zone truckload shipments," IIE Transactions, vol. 39, no. 2, pp. 177-189, 2007. 


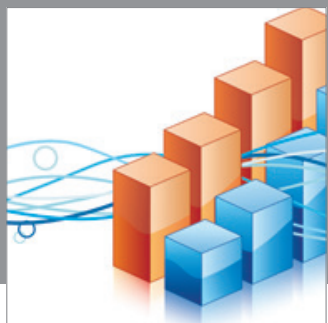

Advances in

Operations Research

mansans

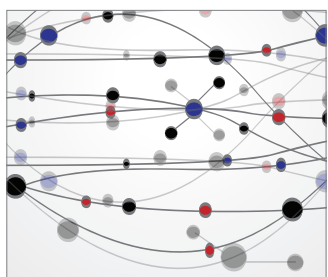

The Scientific World Journal
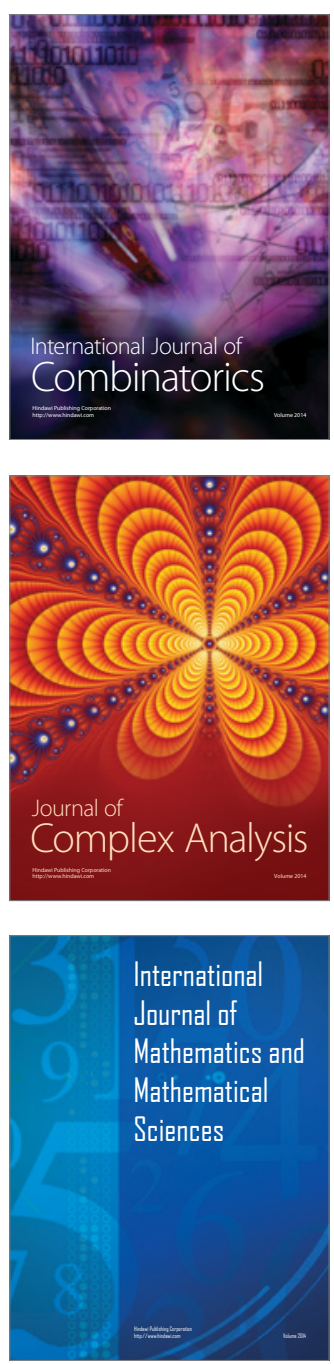
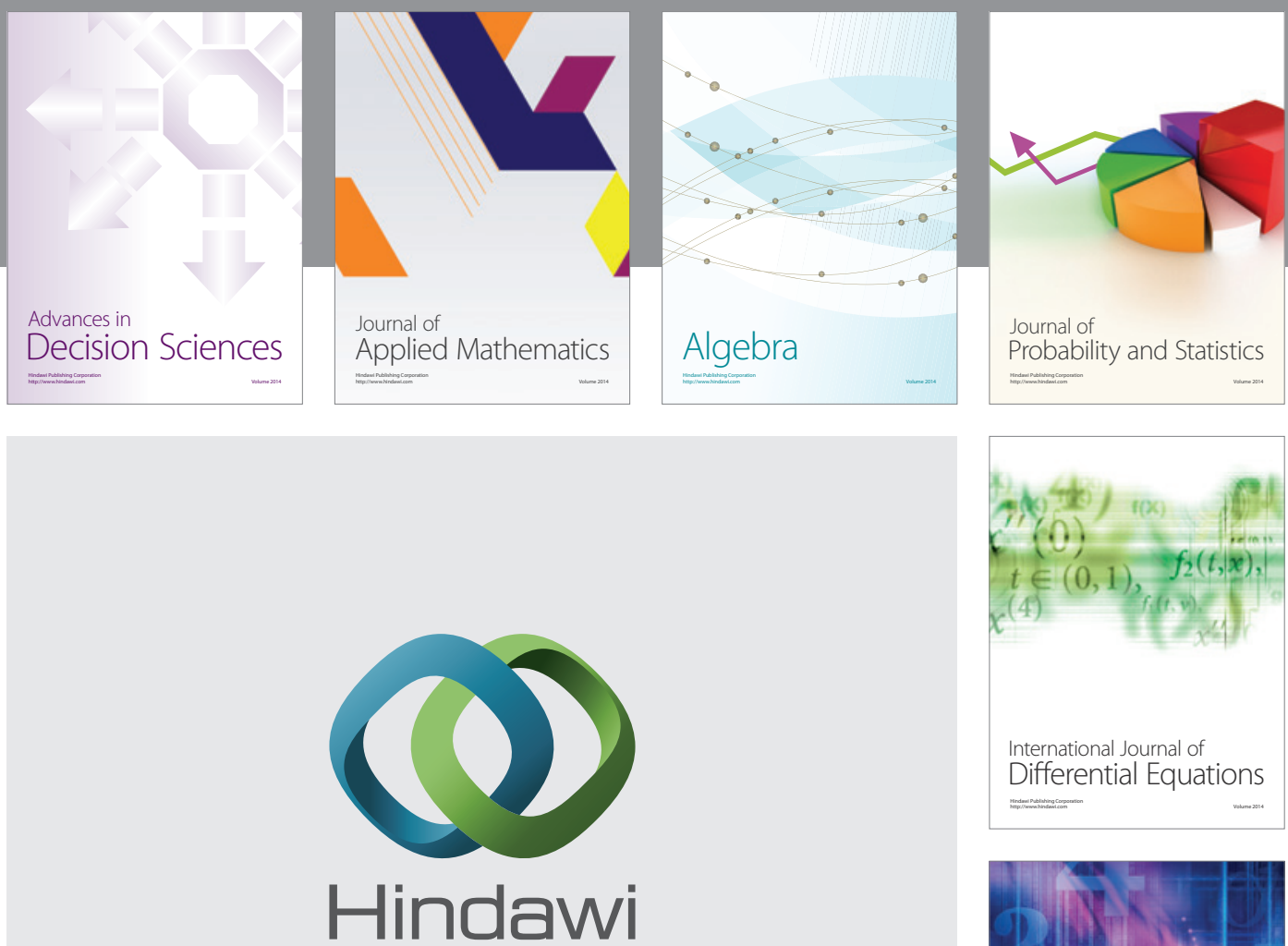

Submit your manuscripts at http://www.hindawi.com
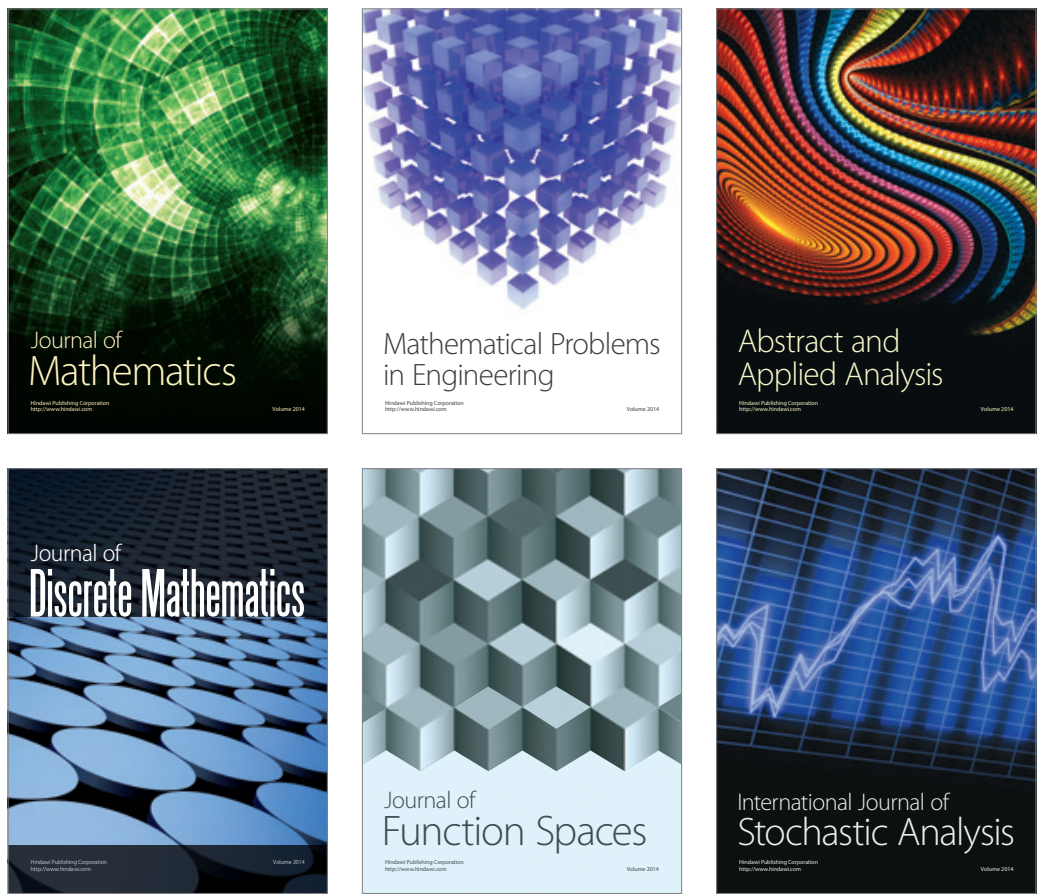

Journal of

Function Spaces

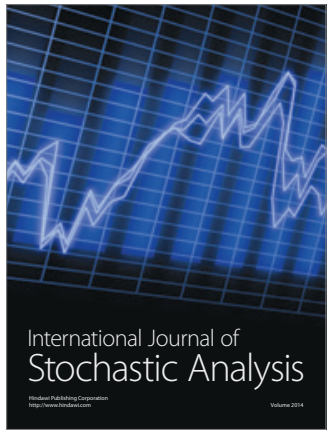

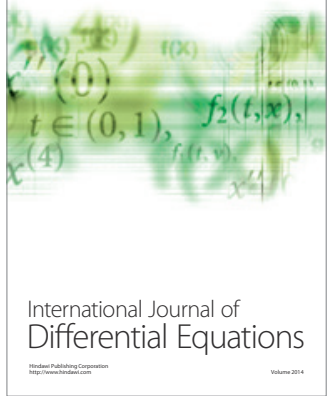
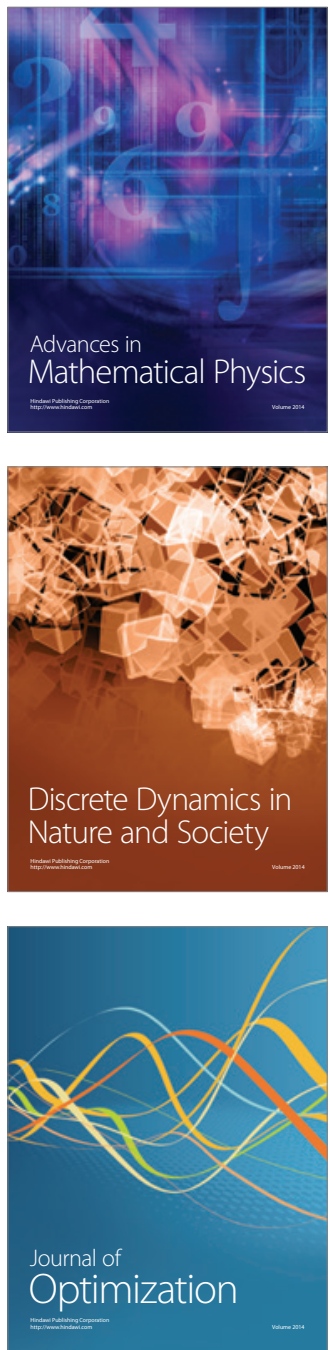